\title{
MODELAGEM E SIMULAÇÃO DO DESEMPENHO DE REATORES DE FLUXO CONTÍNUO E EM BATELADA NO TRATAMENTO DE EFLUENTE TÊXTIL UTILIZANDO O SOFTWARE LIVRE PYTHON.
}

\author{
R.V.SAWAKI ${ }^{1}$, T. C. PARENTE ${ }^{1}$, J.E.C. ALEXANDRE², A.C. LIMA ${ }^{3}$, J.P. RIBEIRO ${ }^{3}$, \\ E.F.ABDALA NETO ${ }^{2}$, R.F. NASCIMENTO ${ }^{3}$ \\ ${ }^{1}$ Universidade Federal do Ceará, Departamento de Engenharia Química \\ ${ }^{2}$ Universidade Federal do Ceará, Departamento de Engenharia Hidráulica e Ambiental \\ ${ }^{3}$ Universidade Federal do Ceará, Departamento de Química Analítica \\ E-mail para contato: rafa3103@gmail.com
}

\begin{abstract}
RESUMO - A modelagem e simulação de processos está cada vez mais presente, devido a sua sofisticação para encontrar soluções para problemas complexos e a possibilidade de validação de projetos, de acordo com a previsão dos resultados. Neste trabalho, tratamos de modelar e simular a eficiência de reatores de fluxo contínuo para o tratamento de efluentes têxteis frente a eficiência de um reator batelada tanto em escala de laboratório quanto em escala industrial (scale up) utilizando o software livre iPython Notebook v3.4.
\end{abstract}

\section{INTRODUÇÃO}

A agressividade ao meio ambiente vem aumentando progressivamente à medida que ocorre o acelerado crescimento populacional e o aumento da atividade industrial. Dentro deste contexto, o setor têxtil apresenta especial destaque, devido a elevada demanda de água exigida em seus processos e a consequente geração de grandes volumes de efluentes. (MEDEIROS, 2011) Desse modo, a Fábrica de Redes Isaac, localizada em Serrinha - CE, estabelece parceria com a Universidade Federal do Ceará em busca do tratamento adequado ao efluente gerado em seu processo, produzindo em torno de vinte mil litros de efluente por dia.

A simulação de processos vem ganhando espaço, devido a sua capacidade de apresentar soluções cada vez mais sofisticadas de problemas cada vez mais complexos encontrados em diversas áreas de estudo, além da simplicidade de uso e sofisticação dos ambientes de desenvolvimento de modelos computacionais, vinculado ao crescimento exponencial do poder de processamento das máquinas. A simulação de processos é definida como a representação de um sistema real explicado por meio de uma modelagem matemática que produz um modelo computacional capaz de entender o comportamento desse sistema, gerando vários cenários experimentais, e de analisar estratégias para sua operação.

Atualmente, a construção e operação de plantas industriais são baseadas principalmente na experiência dos responsáveis pelo seu projeto, por isso a importância da aplicação da modelagem matemática em sistemas reais e simulação de vários cenários de comportamento desses sistemas. O scale up de reatores é um passo importante e essencial na construção e optimização de plantas industriais, e esse termo é definido como "o desenho de um piloto ou 
um reator industrial capaz de replicar através de uma metodologia padrão os resultados obtidos em laboratório", porém há poucas metodologias padrões, sendo a experiência, como já mencionado, a principal ferramenta para desenvolvê-lo, o que acarreta inúmeras dificuldades e erros durante e após a operação de planejamento.

O objetivo deste trabalho é simular o tratamento do efluente têxtil em fluxo contínuo em escala de bancada e em escala industrial, neste último é realizado o scale up tomando como base o reator em batelada utilizado durante os experimentos laboratoriais.

\section{MODELAGEM E SIMULAÇÃO}

O percentual de remoção de DQO foi analisado a partir de um estudo de cinética de degradação para os tempos experimentais. Para tal, utilizou-se o software R versão 3.0.3 pelo método do ajuste de regressão não linear. Os dados de cinética foram então inseridos nos balanços de massa para cada reator CSTR, realizando a simulação do processo no software iPython Notebook versão 3.4.2. Desse modo, foi calculado o volume necessário de reator em fluxo contínuo, em escala de laboratório, no mesmo tempo de operação que o reator em batelada (60 minutos) e comparou-se os valores. Comprovada as vantagens do CSTR, foi realizado o scale up a partir do tempo de tratamento em batelada e obtido o dimensionamento do reator em fluxo contínuo baseando-se nas dimensões do reator em batelada. Desse modo, foi calculado o volume necessário de reator em fluxo contínuo para atender a demanda da fábrica de redes $(13,88 \mathrm{~L} / \mathrm{min})$.

A modelagem do reator CSTR em escala de bancada foi realizada tomando como base a concentração de oxigênio consumido nas amostras coletadas. Desse modo, aplicou-se um balanço de massa para cada reator, equação (1), juntamente com o valor da constante de velocidade de reação no software iPython Notebook 3.4.2. As condições iniciais utilizadas foram a DQO no efluente bruto e o tempo inicial. Toma-se como objetivo a obtenção de uma DQO menor que $200 \mathrm{mg} / \mathrm{L}$, valor predito pela portaria 154/2002 da SEMACE (Superintendência Estadual do Meio Ambiente do Ceará).

$\frac{d C_{A n}}{d t}=\frac{\varphi * C_{A n-1}-{ }^{\varphi} * C_{A n}}{V_{n}}-k * C_{A n}^{2}$

As hipóteses usadas para esta simulação foram: mistura homogênea; regime transiente (concentração varia com o tempo) e escoamento turbulento; vazão de entrada e saída iguais em todos os reatores; e, mesmas condições iniciais de entrada em todos os reatores. A modelagem do reator CSTR em escala industrial possui as mesmas condições que o de bancada e para seu dimensionamento utilizou-se os seguintes critérios: semelhança geométrica (com impelidor na metade da altura de líquido) e manutenção do mesmo nível de agitação.

\section{RESULTADOS E DISCUSSÃO}

Os dados experimentais foram então tratados pelo método de ajuste de regressão não linear obtendo um modelo de cinética de segunda ordem. O valor da constante de velocidade 


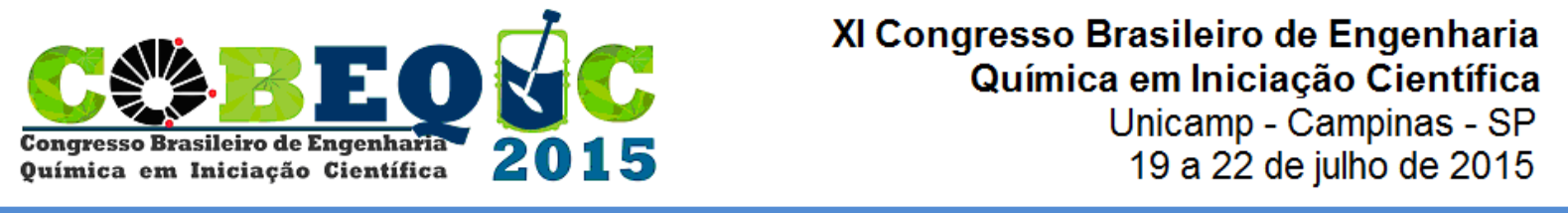

de reação $\mathrm{k}$ obtido foi de $0,0001504726 \mathrm{~L} \mathrm{mg}^{-1} \mathrm{~min}^{-1}$ ou $\mathrm{k}=4,8 \mathrm{~L} \mathrm{~mol}^{-1} \mathrm{~min}^{-1}$ mostrado na figura 2, gerado pelo software R 3.0.3.

Figura 2 - Gráfico do modelo de ajuste de regressão não linear.

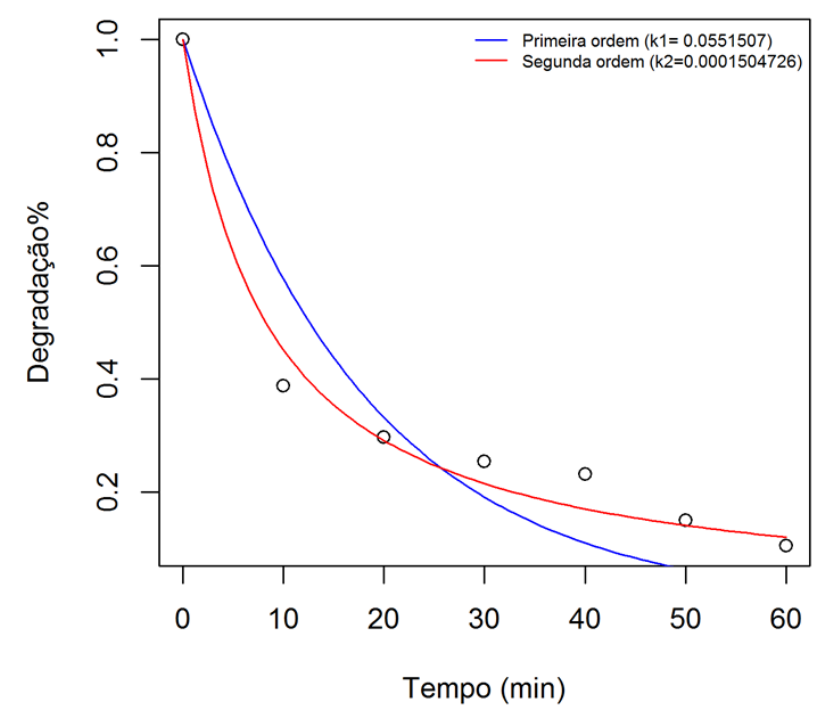

Pelas simulações para o reator CSTR em escala de bancada, chegou-se à conclusão que seriam necessário três CSTR's em série de 4,5L cada (total de 13,5 litros tratados) para se atingir a DQO necessária. O tempo de residência em cada reator foi de 13,63 minutos e o tempo de tratamento da efluente de 44 minutos. A figura 3 mostra um gráfico com as curvas de DQO para cada CSTR e o ajuste dos pontos experimentais do processo em batelada em escala laboratorial.

Figura 3 - Gráfico de DQO experimental e teórica.

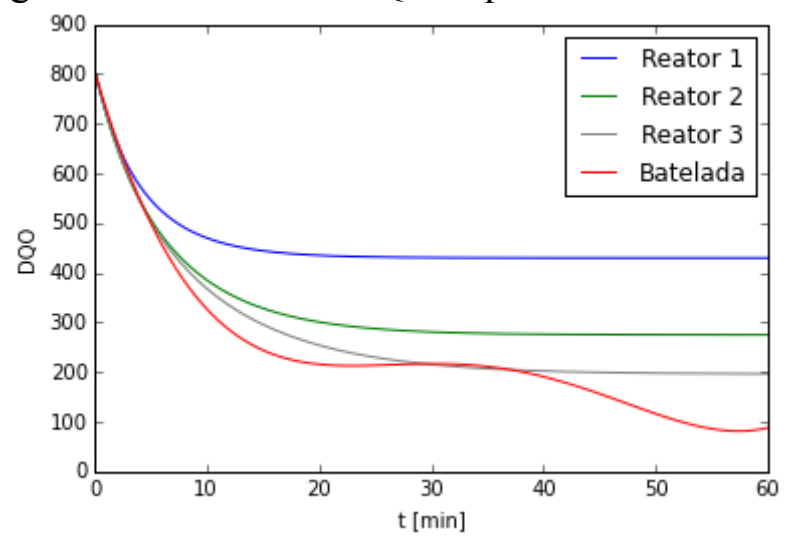

De acordo com a curva experimental, o tempo de tratamento em batelada seria de aproximadamente 39 minutos, tratando 2,7L de efluente, o que há uma diferença de 5 minutos para um volume de tratamento cinco vezes menor.

Para o scale up do CSTR, encontrou-se que seria necessário também uma sequência de necessários dois reatores de 385 litros de volume útil para obter uma DQO de 199,76 mg O 
por litro, utilizando a vazão de $20000 \mathrm{~L} /$ dia ou $13,9 \mathrm{~L} / \mathrm{min}$. O tipo de impelidor escolhido foi a turbina de Rushton, pois oferece mistura de forma axial, ideal para processos em que não há limitações quanto ao cisalhamento. Aplicando um fator de segurança de $25 \%$, obtém-se um volume total de 510 litros para cada reator. Sabendo o volume necessário e o tempo de operação do processo em batelada, foi realizado o scale up utilizando as equações explanadas abaixo. O subscrito 1 indica a dimensão do modelo e o subscrito 2 indica a dimensão do protótipo.

$\left({ }^{T} /{ }_{D}\right)_{1}=\left({ }^{T} / D\right)_{2}$

$\left({ }^{H} /\right)_{1}=\left({ }^{H} / D\right)_{2}$

$(h / D)_{1}=(h / D)_{2}$

$\left({ }^{B} / D\right)_{1}=\left({ }^{B} /\right)_{2}$

$R e_{\text {impelidor }}=\frac{N D^{2}}{v}$

$\left(N_{A}\right)_{1}=\left(N_{A}\right)_{2}$

$\left(\frac{\dot{W}_{u}}{V_{l}}\right)_{1}=\left(\frac{\dot{W}_{u}}{V_{l}}\right)_{2}=N_{A}$

$V_{\text {líquido }}=V_{l}=\frac{\pi T^{2}}{4} H$

$\dot{W}_{u}=\rho \cdot N^{3} \cdot D^{5} \cdot N_{P O}$

Margem de segurança para o volume

A figura 4 mostra um gráfico com as curvas de DQO para cada CSTR e o ajuste dos pontos experimentais do processo em batelada em escala laboratorial.

Figura 4 - Gráfico de DQO experimental e teórica.

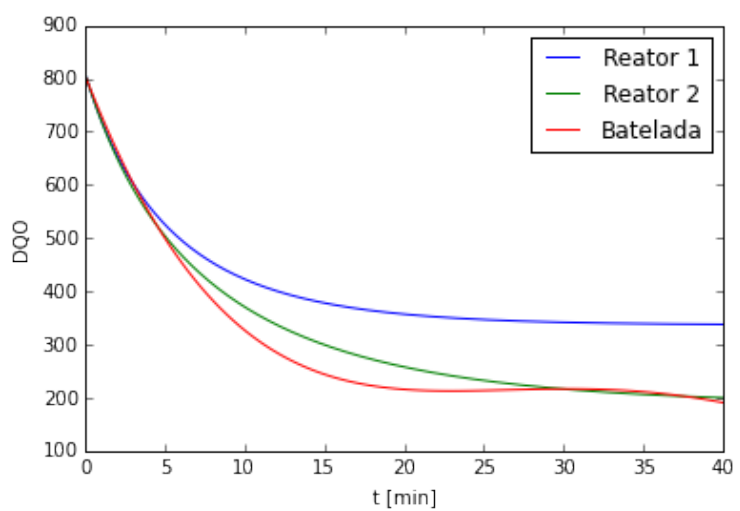


Os resultados obtidos nas equações estão na tabela 1 e a ilustração do reator está na figura 5. Os eletrodos utilizados no sistema em batelada foram dimensionados como chichanas para o reator em fluxo contínuo simulado, sendo posicionados na lateral do reator.

Tabela 1 - Resultados obtidos para reator em escala industrial.

\begin{tabular}{cccc}
\hline Dimensões & $\begin{array}{c}\text { Batelada } \\
(\mathrm{cm})\end{array}$ & $\begin{array}{c}\text { Contínuo } \\
(\mathrm{cm})\end{array}$ & $\begin{array}{c}\text { Aumento } \\
(\%)\end{array}$ \\
\hline $\mathrm{T}$ & 10 & 50,78 & 507,81 \\
$\mathrm{H}$ & 37,43324 & 190,09 & 507,81 \\
$\mathrm{~h}$ & 18,71662 & 95,05 & 507,81 \\
$\mathrm{~B}$ & 5 & 25,39 & 507,81 \\
$\mathrm{D}$ & 5 & 8,89 & 177,73 \\
$\mathrm{H}-\mathrm{h}$ & 18,71662 & 95,05 & 507,81 \\
\hline
\end{tabular}

Figura 5 - Ilustração do reator contínuo simulado

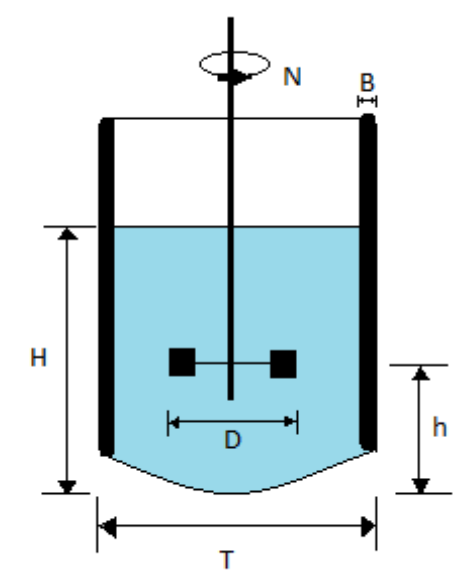

\section{ANALISE ECONÔMICA}

A técnica de eletrocoagulação flotação utilizando corrente contínua pulsada é conhecida por seu baixo custo. Para a escala em bancada, podemos calcular a potência, em Watts, consumida através da equação 12. A tensão para a cidade de Fortaleza é de 220 volts e a corrente empregada no trabalho é 0,377 ampères. Com isso, multiplica-se pelo tempo experimental, em horas, de operação necessário para obter DQO de $200 \mathrm{mgO}_{2} / \mathrm{L}$ e depois divide-se pelo volume de efluente tratado. Desse modo temos um resultado de 21,27 e 4,49 $\mathrm{kWh} / \mathrm{m}^{3}$ para o reator em batelada e o CSTR, respectivamente. De acordo com a companhia energética do Ceará (COELCE), o valor do $\mathrm{kW} / \mathrm{h}$ é de $\mathrm{R} \$ 0,29815$. Assim, conclui-se que temos um custo de operação no valor de $\mathrm{R} \$ 6,09$ e $1,34 / \mathrm{m}^{3}$ para o reator em batelada e o CSTR, respectivamente.

$P=U * i$ 


\section{NOMENCLATURA}

\begin{tabular}{|c|c|c|c|c|c|}
\hline $\begin{array}{l}\text { B - Largura } \\
\text { da chicana. }\end{array}$ & $\begin{array}{l}\text { D - Diâmetro } \\
\text { do impelidor }\end{array}$ & $\begin{array}{c}\text { k - Constante } \\
\text { de velocidade } \\
\text { da reação. }\end{array}$ & $\begin{array}{c}\rho- \\
\text { Densidade da } \\
\text { água. }\end{array}$ & $\begin{array}{l}\text { U - Tensão } \\
\text { aplicada na } \\
\text { eletrólise. }\end{array}$ & $\begin{array}{l}\text { Vl, Vn- } \\
\text { Volume } \\
\text { do líquido } \\
\text { útil. }\end{array}$ \\
\hline $\begin{array}{l}\text { Can - } \\
\text { Concentração } \\
\text { do reagente. }\end{array}$ & $\begin{array}{c}\mathrm{h}-\text { Altura } \\
\text { entre o } \\
\text { impelidor e o } \\
\text { fundo do } \\
\text { reator. }\end{array}$ & $\begin{array}{l}\mathrm{Na} \text { - Nível de } \\
\text { agitação. }\end{array}$ & $\begin{array}{l}\text { Re impelidor } \\
\text { - Número de } \\
\text { Reynolds do } \\
\text { impelidor. }\end{array}$ & $\begin{array}{c}\varphi \text { - Vazão de } \\
\text { entrada e } \\
\text { saída. }\end{array}$ & $\begin{array}{l}\text { Wu- } \\
\text { Potência } \\
\text { útil. }\end{array}$ \\
\hline i-Corrente. & $\begin{array}{c}\mathrm{H}-\text { Volume } \\
\text { útil. }\end{array}$ & $\begin{array}{l}\mathrm{N} \text { - Número } \\
\text { de rotação do } \\
\text { impelidor. }\end{array}$ & $\begin{array}{c}\mathrm{T} \text { - Diâmetro } \\
\text { do reator. }\end{array}$ & $\begin{array}{c}v- \\
\text { Viscosidade } \\
\text { da água. }\end{array}$ & \\
\hline
\end{tabular}

\section{CONCLUSÕES}

O objetivo de comparação entre o reator contínuo e batelada foi alcançado. Viu-se que em 60 minutos atinge-se a DQO com valor inferior a 200 ppm $\mathrm{O}_{2}$ para 13,5 litros de efluente enquanto que, no batelada, para esse mesmo tempo tratam-se 2,7 litros, além de que o gasto energético para o tratamento foi de 4,54 vezes menor para o CSTR. O volume requerido pela fábrica para operar o reator em batelada seria de 20000 litros, enquanto que no sistema contínuo industrial, esse volume foi diminuído para 770 litros para um tempo de 40 minutos que seria o mesmo tempo do batelada real de 2,7L. Valor mais viável para instalação da estação de tratamento de efluente (ETE).

Embora o investimento inicial em sistemas contínuos seja maior, normalmente os custos operacionais por volume de efluente tratado são menores. Fato que leva a preferência por reatores de sistema contínuo quando a capacidade de processamento requerida é grande.

Para a validação do modelo do CSTR em bancada, seria necessário fazer o tratamento do efluente têxtil em laboratório do modo como foi proposto e a medição da potência do impelidor para completar o cálculo do gasto energético total.

\section{REFERÊNCIAS}

MEDEIROS, A. G. Avaliação dos tratamentos convencional e por oxidação química na degradação de corantes em efluentes têxteis. Dissertação (Mestrado em Engenharia de Processos). Universidade da região de Joinville, UNIVILLE, 2011. 